\title{
Extending Navigation Modelling to Support Content Aggregation in Web Sites*
}

\author{
Pedro Valderas, Joan Fons, and Vicente Pelechano \\ Department of Information Systems and Computation \\ Polytechnic University of Valencia \\ Camí de Vera s/n \\ 46022 Valencia, Spain \\ \{pvalderas, jjfons, pele\}@dsic.upv.es
}

\begin{abstract}
Currently, web sites are integrators of heterogeneous information and services that are oriented to providing content aggregation. From a ModelDriven perspective, web applications need conceptual mechanisms that make it easy to describe, manage and reuse contents and services in order to deal with content aggregation at a higher level of abstraction. Our work presents conceptual modelling techniques that extend the OOWS navigational modelling by refining the navigational context definition and introducing the concept of information abstraction unit to specify the contents of web applications of this kind. These new abstractions provide powerful reuse mechanisms that produce considerable benefits because both development time and effort can be reduced. Finally, the paper presents some ideas to implement content aggregation taking these enhanced navigational models as input.
\end{abstract}

\section{Introduction}

Web sites are assuming a greater leading role in Internet. Web sites like Yahoo (www.yahoo.com), MSN (www.msn.com), Lycos (www.lycos.com) or Terra (www.terra.es) are ranked among the web applications that receive the most visits. The main characteristic of applications of this type consists of providing the users with web pages that are built from aggregation of contents. These pages provide a lot of information and services on issues of a diverse nature. Figure 1 shows the home page of the Terra web site that is build from the aggregation of diverse contents or information blocks.

From a methodological point of view, the most outstanding approaches (OOHDM [2], WebML [5], WSDM [1], etc.) focus their efforts on defining web applications from conceptual models that allow them to specify hypermedial and functional requirements. The mechanisms for hypermedia modelling allow the analyst (1) to define web pages as conceptual schema views and (2) to interconnect these views to define the navigational structure of the web application. However, considering a web

* This work has been partially supported by the MCYT Project with ref. TIC2001-3530-C0201 and the Polytechnical University of Valencia, Spain. 
page only as a view of the conceptual schema makes difficult to specify aggregation of contents, where web pages are built as a composite of several conceptual schema views (see each numbered area in Figure 1). In this way, although some of the approaches mentioned above provide support in the design and/or implementation steps, none of them explicitly supports the specification of web applications with aggregation of contents in the conceptual modelling step.

In this work, we present conceptual modelling techniques that extend the OOWS (ObjectOriented Web Solution) [4] navigational modelling by refining the navigational context definition and introducing the concept of information abstraction unit in order to specify the contents for web applications of this kind. This new expressive capacity makes it easy to describe at a higher level of abstraction of web applications with content aggregation. It also provides powerful reuse mechanisms that

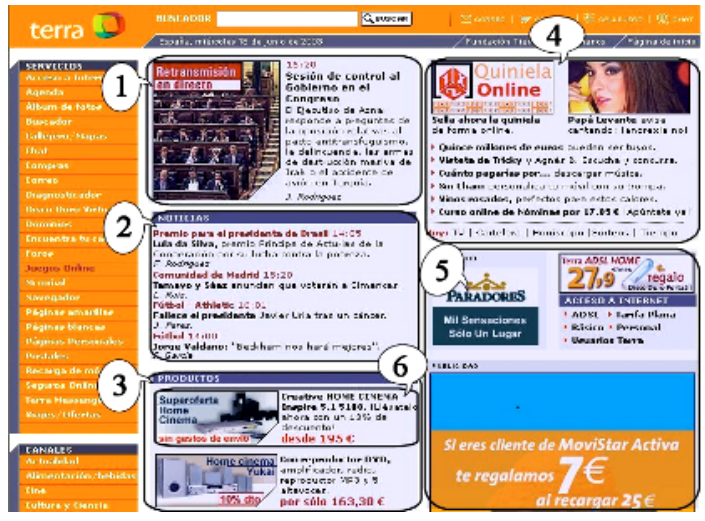

Fig. 1. Terra Home Page can reduce development time and effort. Following a Model-Driven Development (MDD [6]) approach, the paper presents some ideas to implement web applications that support content aggregation by taking these enhanced navigational models as input.

This paper is organized in the following way: section 2 introduces the OOWS method which presents the proposed extensions at a conceptual modelling level using the Terra web site as a case study. Conclusions and future works are commented on in section 3 .

\section{Content Aggregation in OOWS}

OOWS (Object-Oriented Web Solutions) [3] is the extension of an object-oriented software production method (OO-Method [4]) that introduces the required expressivity to capture the navigational requirements of web applications. In order to do this, OOWS introduces the Navigational Model that allows for capturing the navigation semantics in two steps: the "Authoring-in-the-large" (global view) and the "Authoring-in-the-small" (detailed view).

The Authoring-in-the-large step refers to the specification and design of global and structural aspects of the web application. These requirements are specified in a Navigational Map that provides a specific kind of user with its system view. It is represented using a directed graph whose nodes denote navigational contexts and whose arcs denote navigational links or valid navigational paths.

These navigational contexts (graphically represented as UML packages stereotyped with the «context» keyword) represent the user interaction units that provide a set of 
cohesive data and operations. The navigational links (navigational map arcs) represent context accessibility or "navigational paths". Figure 2 shows a piece of the navigational map for the Terra web site related to an anonymous Internet user.

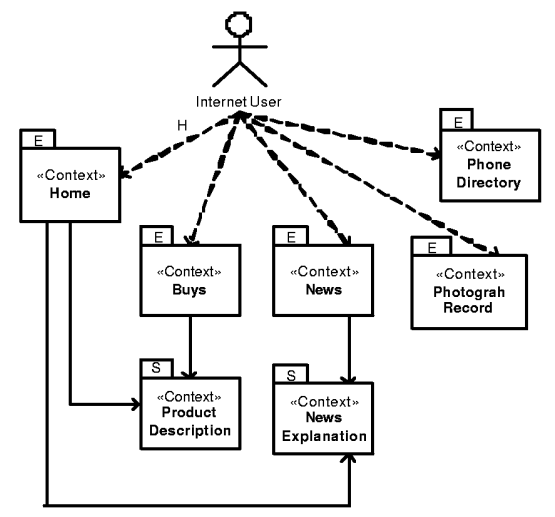

Fig. 2. Terra navigational map

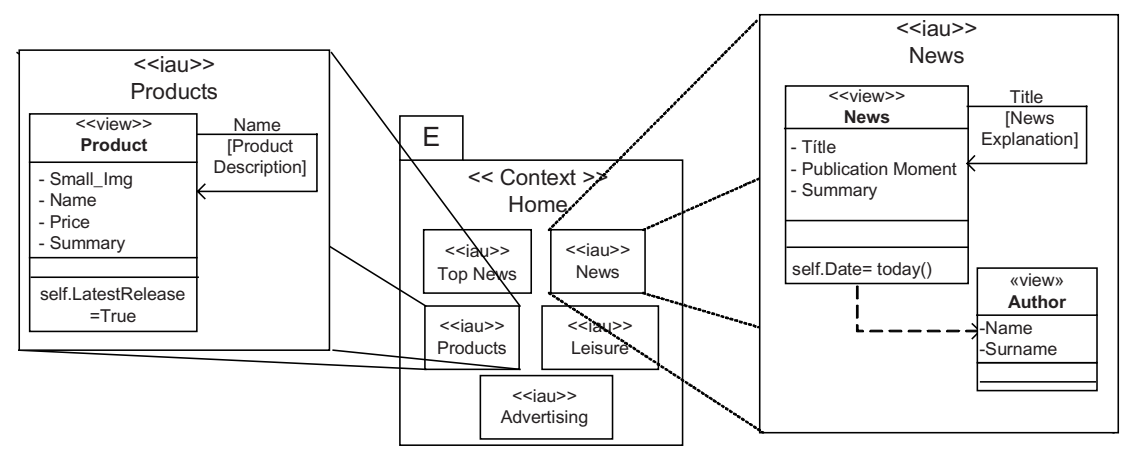

Fig. 3. Home navigational context and News and Products IAUs

The "Authoring-in-the-small" step refers to the detailed specification of the contents of the navigational contexts. The following section presents how these contexts must be defined in order to achieve content aggregation.

\subsection{Navigational Contexts with Content Aggregation}

In order to support content aggregation, navigational contexts should be considered as user interaction units which provide access to several information abstraction units. An information abstraction unit (IAU), stereotyped with the «iau» keyword, represents a specific view on the class diagram. Each IAU is made up of a set of navigational classes that represent class views (including attributes and operations). These classes are stereotyped with the «view» keyword. Moreover, selection filters (expressions that allow us to select specific objects) can be defined in OCL upon an 
object population which has been retrieved by a navigational class (see Figure 3, "self.latestRelease=True" at Products class and "self.Date=today()" at News class).

Navigational classes must be related by unidirectional binary relationships, called navigational relationships. They are defined over existing aggregation/association/ composition or specialization/generalization relationships.

Figure 3 shows an example of the Home navigational context. This context is defined using five IAUs: Top News, News, Products, Leisure and Advertising. Figure 3 also shows the News IAU definition (that provides the news of the day) and the Products IAU definition (that provides information about the latest product releases). In Figure 1 we can see the implementation of this navigational context. In areas 1, 2, 3, 4 and 5 we can see the IAUs implementation corresponding each one to a different section of the home page.

\subsection{Reuse Mechanisms}

Introducing IAUs into the OOWS navigational model allows us to provide some mechanisms to reuse contents at a high level of abstraction. These mechanisms allow us (1) to specify new navigational contexts and/or (2) to specify new IAUs taking a predefined IAU as an input.

1. Specifying New Navigational Contexts: an IAU specified in a navigational context can be used to define other new contexts by means of a reference to it in the new definition. In this way, a new navigational context can be made up of: (1) new IAUs (mandatory option for the navigational context which is specified first) or (2) IAUs specified in other navigational contexts.

2. Specifying New IAUs through specialization: new IAUs can be defined taking an already defined one as a basis. We do this by extending the IAU definition by

means of specialization mechanisms: the specialized IAU inherits a parent IAU definition. It can be refined

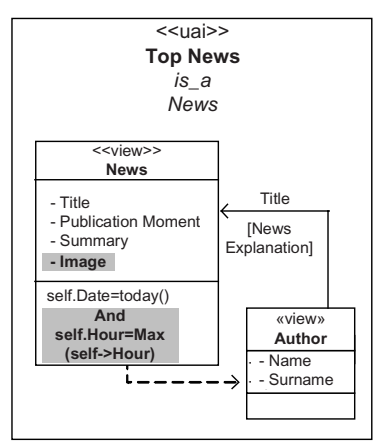

Fig. 4. Top News IAU

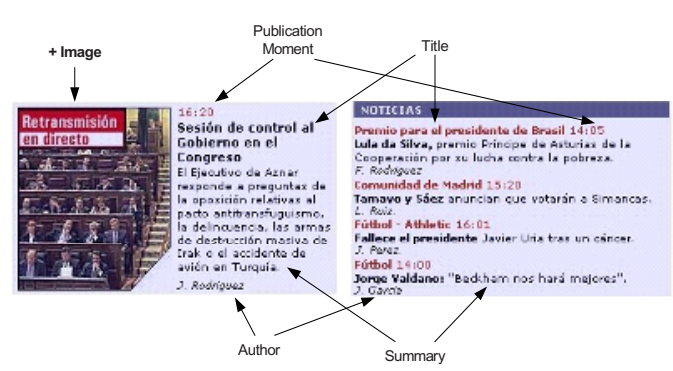

Fig. 5. Top News and News IAUs implementation.

to adapt the retrieved information, services and navigation capabilities to the needs of a particular navigational context, carrying out the following operations: Adding or removing attributes, adding or removing services, adding or removing navigational classes, adding or removing navigational relationships and adding, removing or redefining population selection filters.

IAU specialization is performed by means of some kind of is_a operator, allowing the analyst to refine the specialized IAU using the previous operations. Figure 4 shows the Top News IAU definition. This IAU has been specialized from the News IAU (see the top of the Figure 4). In this 
case, it has been refined by adding the Image attribute and redefining its population selection filter (only the last news item is retrieved at this moment). These refinements have been highlighted in Figure 4. Figure 5 shows sections 1 and 2 of Figure 1 corresponding to the implementation for both Top News and News IAUs.

\section{Conclusions}

In this paper we have proposed a solution from the conceptual modelling perspective in order to give methodological support for the development of web applications with content aggregation.

This solution redefines the navigational context as a conceptual abstraction that can be built using multiple information abstraction units (IAUs). Each IAU represents at the conceptual modelling step a specific content and the use of IAUs allows us to carry out content reuse mechanisms.

As future work, it would be necessary both to extend the presentation patterns that OOWS Presentation Model provides to attach them to the IAUs and to detect presentation relationships and dependencies between different IAUs in the same navigational context.

\section{References}

1. De Troyer O. and Leune C. WSDM: A user-centered design method for Web sites. In Proc. of the 7th International World Wide Web Conference, 1998.

2. Schwabe D., Rossi G., "An Object Oriented Approach to Web-Based Application Design", Theory and Practice of Object Systems 4(4), 1998. Wiley and Sons, New York, ISSN 1074-3224).)

3. Joan Fons, Vicente Pelechano, Manoli Albert y Oscar Pastor. Development of Web Applications from Web Enhanced Conceptual Schemas. Springer-Verlag, Lecture Notes in Computer Science. Proc. Of the International Conference on Conceptual Modelling, 22nd Edition, ER'03, pp 232-245. Chicago, EE.UU, 13 - 16 October 2003.

4. Pastor O., Pelechano V., Insfrán E,. and Gómez J. From Object Oriented Conceptual Modeling to Automated Programming in Java. 17th International Conference on Conceptual Modeling (ER'98). Springer-Verlag, LNCS 1507, pp. 183-196. Singapore, November, 1998.

5. Ceri S., Fraternali P., Bongio A. Web Modeling Language (WebML): a Modeling Language for Designing Web Sites. In WWW9, Vol. 33 (1-6), pp 137-157. Computer Networks, 2000.

6. Mellor, S.J.; Clark, A.N.; Futagami, T. "Model-driven development" - Guest editor's introduction. IEEE Software, p. 14-18, Sept.-Oct. 2003. 